\title{
Screening for breast cancer
}

Harvard Medical School, Boston, MA, USA

Evidence about screening for breast cancer demonstrates how principles of clinical epidemiology can be used to aid in decisions about clinical care and health care policy. Regardless of condition, three key questions must be asked and answered before undertaking screening: 1) How great is the burden of suffering caused by the condition? 2) How effective is early treatment following screening (as opposed to results of treatment when the condition would be diagnosed without a screening program)? and 3) How good is the screenning test in terms of sensitivity, specificity, predictive value, simplicity, cost, safety, acceptability and labeling effects?

Breast cancer incidence and mortality varies by country. In the United States, breast cancer is the most common non-skin cancer and second most deadly cancer in women. There are three main tests used, either alone or in combination, for breast cancer screening: mammography, clinical breast examination by a health professional, and breast selfexamination.

Mammography, with or without clinical breast examination clearly reduces mortality in women aged 50-69, but the effect in younger women remains unclear even after trials involving approximately 170,000 women. Eight randomized controlled trials all showed mortality reductions for women over 50 years of age; at seven years of follow-up, the combined result showed a $34 \%$ mortality reduction that was statistically significant. These same studies were inconsistent in finding mortality reductions in women ages $40-49$, combined analyses showed no effect at seven years of follow-up and a $10 \%$ to $17 \%$ statistically insignific 1 ant reduction at 10 to 12 years. This delayed and smaller trend in younger women may be due to the fact that many women entering trials in their forties had their cancers diagnosed when they were in their fifties, at an age when mammography is known to be effective. Only one randomized controlled trial has been done comparing mortality differences after either mammography or a very through clinical breast examination. At seven years of follow-up, there was no difference in mortality rates. No randomized trials of breast self-examination have reported mortality data.

Mammography is the most sensitive screening test currently available for breast cancer screening. The high falsepositive rate of mammography can lead to substantial numbers of women experiencing anxiety about breast cancer, the possibility of overdiagnosis with "pseudocancers" is real, and the high cost of mammography in the United States means already almost $\$ 3$ billion is spent annualy on mammography, about half or which is for women under age 50 .

When preventive policies are promulgated, strong evidence of benefit is essential because so many people must be screened to potentialy help one person. While much more needs to be done to ensure that all women aged 50-69 receive routine screening in countries where breast cancer on an important health risk, more research is necessary before instituting routine breast cancer screening in younger women. 